\title{
Space and Time in the Nucleus Developmental Control of Replication Timing and Chromosome Architecture
}

\author{
D.M. Gilbert, S.-I. Takebayashi, T. Ryba, J. Lu, B.D. Pope, K.A. Wilson, and I. Hiratani \\ Department of Biological Science, Florida State University, Tallahassee, Florida 32306 \\ Correspondence: gilbert@bio.fsu.edu
}

\begin{abstract}
All eukaryotic cells replicate segments of their genomes in a defined temporal sequence. In multicellular organisms, at least half of the genome is subject to changes in this temporal sequence during development. We now know that this temporal sequence and its developmentally regulated changes are conserved across distantly related species, suggesting that it either represents or reflects something biologically important. However, both the mechanism and the significance of this program remain unknown. We recently demonstrated a remarkably strong genome-wide correlation between replication timing and chromatin interaction maps, stronger than any other chromosomal property analyzed to date, indicating that sequences localized close to one another replicate at similar times. This provides molecular confirmation of long-standing cytogenetic evidence for spatial compartmentalization of early- and late-replicating DNA and supports our earlier model that replication timing is reestablished in each $G_{1}$ phase, coincident with the anchorage of chromosomal segments at specific locations within the nucleus (timing decision point [TDP]). Here, we review the evidence linking the replication program to the three-dimensional architecture of chromatin in the nucleus and discuss what such a link might mean for the mechanism and significance of a developmentally regulated replication program.
\end{abstract}

Connections between replication timing and the three dimensional (3D) organization of chromosomes were first made several decades ago, shortly after the discovery of a defined replication-timing program (Taylor 1958, 1960). For example, it was observed that one of the two female $\mathrm{X}$ chromosomes took on a compact structure termed the Barr body and localized to the periphery of the nucleus coincident with a switch to late replication, whereas its active homolog remained earlier replicating, decondensed, and interiorly localized (Hiratani and Gilbert 2010). Later, it was discovered that replication takes place in spatially distinct compartments of the nucleus at different times during S phase (Nakamura et al. 1986; Nakayasu and Berezney 1989; O'Keefe et al. 1992). Early replication takes place in the interior of the nucleus, whereas the chromatin at the nuclear and nucleolar periphery replicates later in $\mathrm{S}$ phase. This $3 \mathrm{D}$ organization is established during early $\mathrm{G}_{1}$ phase, coincident with the establishment of the replication-timing program (Dimitrova and Gilbert 1999; $\mathrm{Li}$ et al. 2001; Wu et al. 2006; Lu et al. 2010). The presumption has generally been that this spatiotemporal organization is somehow related to the transcriptional functions of chromosomes, because different types of chromatin are assembled at different times during $\mathrm{S}$ phase and most transcribed genes are early replicating, however, the evidence for this is still circumstantial (Hiratani et al. 2009). Recently, genome-wide changes in replication timing have been mapped that correlate significantly but incompletely with changes in transcription, and replication timing has been shown to correlate even more strongly with 3D maps of chromatin interactions, supporting at least an indirect relationship between these structural and functional properties of chromosomes (Hiratani et al. 2008, 2010; Ryba et al. 2010). These changes occur within defined segments along the length of all chromosomes, suggesting that individual autosomal domains experience developmental regulation similar to the facultative heterochromatinization originally observed on inactive mammalian female X chromosomes (Hiratani and Gilbert 2010). In fact, genome-wide studies have been complemented with single-cell analyses of individual loci by in situ hybridization, demonstrating that changes in replication timing are associated with changes in chromatin compaction and subnuclear position (Hiratani et al. 2010; S Takebayashi, unpubl.). Here, we summarize old and new data from our laboratory that links the 3D organization of chromatin to replication timing and then speculate on the biological significance of this liaison.

\section{REPLICATION DOMAINS AND REPLICATION FOCI: STABLE UNITS OF CHROMOSOME STRUCTURE AND FUNCTION}

In eukaryotic cells, DNA replication proceeds via the synchronous firing of spatially clustered replication origins that can be visualized as regions of bidirectional DNA synthesis along the lengths of purified and stretched DNA fibers (Fig. 1) (Jackson and Pombo 1998; Berezney et al. 2000; Gilbert and Gasser 2006). In mammals, these "replicon clusters" or replication domains comprise several hundred kilobases of DNA and replicate in a defined temporal order (Nakamura et al. 1986; Ma et al. 1998). There is a long-standing correlation between early replication and transcriptional activity with chromatin assembled early in $\mathrm{S}$ phase that is consider- 


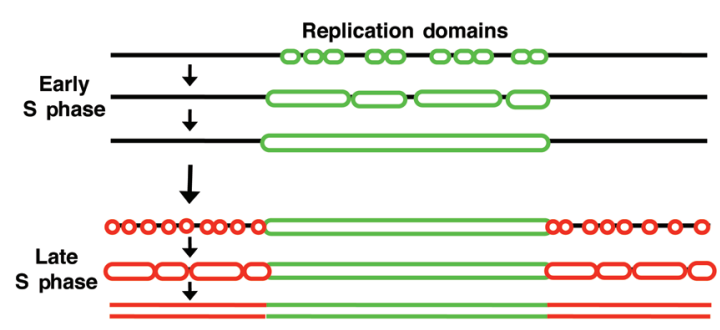

Figure 1. Replication domains. In eukaryotes, replication proceeds in a temporal order defined by the coordinated and nearly synchronous firing of multiple replication origins across hundreds of kilobases (Huberman 1991; Jackson and Pombo 1998; Gilbert and Gasser 2006). For simplicity, adjacent domains are illustrated as replicating either early or late, but there is in fact a continuum of replication times throughout $\mathrm{S}$ phase.

ably more enriched for active histone modifications (Hiratani et al. 2009; Lande-Diner et al. 2009). Recent genomewide mapping of replication timing has confirmed the existence of well-defined molecular boundaries that separate early-replicating from late-replicating domains throughout the genome (Hiratani et al. 2008; Hiratani and Gilbert 2009; Pope et al. 2010), although the boundaries for histone modifications and other epigenetic marks are not as precisely defined (Thurman et al. 2007; Ryba et al. 2010). A correlation with transcription predicts that replication timing should change coordinately with differential gene expression during development, and this is indeed the case. However, the relationship is clearly not direct; many genes change replication timing without a change in transcription and vice versa, although a small number of exceptional genes are transcribed or even induced within late-replicating chromatin (Hiratani et al. 2008). These observations have led to the notion that early replication is generally, with some exceptions for certain classes of genes, a necessary but not sufficient condition for transcription (Hiratani et al. 2009).

How might replication be related to chromatin structure? First, it is not just DNA that replicates; the entire structure of the chromosome must be taken apart and reassembled, and there is limited but compelling evidence that different types of chromatin are assembled at different times during $\mathrm{S}$ phase (Lande-Diner et al. 2009). Thus, replication is both a window of opportunity to study chromatin packaging and an opportunity for the cell to change the structure of chromatin by changing the time during $\mathrm{S}$ phase at which chromatin is assembled. There is also substantial evidence that chromatin influences replication timing, presumably by controlling the access of replication proteins to initiation factors (Bickmore and Carothers 1995; Vogelauer et al. 2002; Goren et al. 2008), and direct evidence for the regulation of replication timing by the heterochromatin protein Swi6 has recently been obtained in fission yeast (Hayashi et al. 2009). These two concepts can be merged into an attractive model in which the chromatin structures assembled in one cell cycle dictate the time of replication in the next cell cycle, providing a selfpropagating memory of epigenetic states (Gilbert 2002). Also, because replication-timing changes are regulated at the level of large domains, changes in replication timing could rapidly propagate a change in chromatin structure across hundreds of kilobases. This model is attractive but remains speculative. Unfortunately, we know very little about what regulates the replication-timing program, so there has been limited means with which to manipulate this program to probe causal relationships.

Early- and late-replicating domains reside in different compartments within the nucleus that can be visualized by pulse labeling with nucleotide analogs and by staining with fluorescent reagents that reveal the location of active DNA synthesis (Nakamura et al. 1986; Nakayasu and Berezney 1989; O’Keefe et al. 1992). Labeled sites appear as spots in the nucleus known as replication foci. These foci can also be observed directly in living cells by labeling with fluorescent nucleotides or replication fork proteins, showing that they are not artifacts of fixation (Manders et al. 1999; Leonhardt et al. 2000; Sadoni et al. 2004; Panning and Gilbert 2005). In all cell types from multicellular species examined to date, early replication takes place in the interior of the nucleus throughout the first half of S phase, and there is always a dramatic transition to more peripherally localized foci near the middle of S phase, making it very tempting to speculate that this represents a transition from euchromatin to facultative heterochromatin replication. The very end of S phase is generally devoted to the replication of large blocks of heterochromatin, although some species-specific differences in the timing of constitutive heterochromatin, defined by trimethylation of histone $\mathrm{H} 3$ lysine 9, have been identified (Wu et al. 2005). Interestingly, when pulse-labeled replication foci are chased into subsequent cell cycles, the labeled segments do not mix, separate, or change in shape, size, or intensity, suggesting that the DNA that replicates together remains a stable structural and functional unit of interphase chromosome structure (Jackson and Pombo 1998; Gilbert and Gasser 2006; Maya-Mendoza et al. 2010). However, replication foci are cytogenetically defined entities; a question of major interest is whether replication domains defined by genome-wide studies are the molecular equivalent to replication foci.

\section{THE REPLICATION TIMING DECISION POINT}

How might 3D organization be related to replication timing? More than 10 years ago, we developed a system in which we first tagged early- and late-replication domains with different halogenated nucleotides in one cell cycle and then prematurely jump-started $\mathrm{S}$ phase at different times during $G_{1}$ phase of the following cell cycle by introducing nuclei from these cells into a cell-free replication system derived from Xenopus eggs (Dimitrova and Gilbert 1999). Extracts from Xenopus eggs can initiate replication rapidly and synchronously within nuclei isolated at any time during $G_{1}$ phase, allowing us to evaluate the preparedness of chromatin within these nuclei for executing their normal replication program. We found that, with nuclei isolated shortly after mitosis, replication took place in a random temporal order, whereas at a discrete point during $\mathrm{G}_{1}$, the nuclei experienced a transition that could dictate the proper 


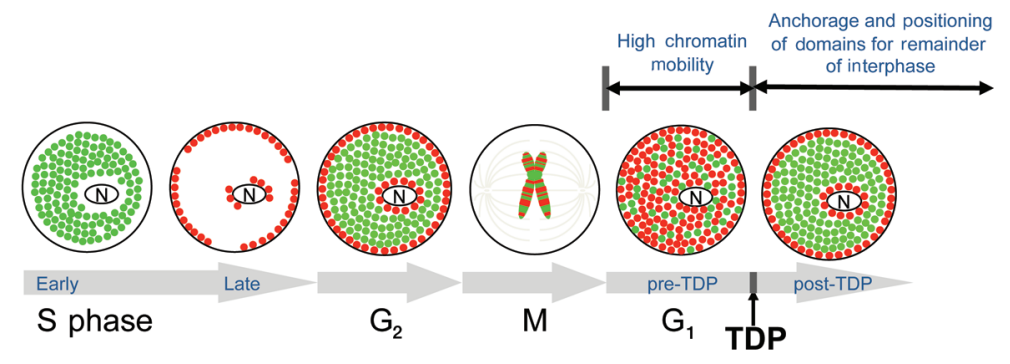

Figure 2. Spatial reorganization of chromatin and replication timing is established at the TDP. Diagram illustrates experimental scheme used to identify the TDP (Dimitrova and Gilbert 1999). Replication domains located in the nuclear interior were labeled with one nucleotide analog early during S phase, and the perinuclear and perinucleolar replication domains were labeled with a different nucleotide analog late during S phase. Labeled cells were then chased into mitosis, during which these differential labels could be visualized as $\mathrm{R}$ and $\mathrm{G}$ bands, respectively, on mitotic chromosomes. Synchronous populations of mitotic cells were released into $\mathrm{G}_{1}$ phase, and positions of early- and late-replication domains were monitored, identifying the time during early $\mathrm{G}_{1}$ phase at which they returned to their stable interphase positions. When nuclei from these same $\mathrm{G}_{1}$-phase cells were introduced into a Xenopus egg extract to initiate DNA replication, replication took place in a random temporal sequence within nuclei before domain repositioning but in the proper temporal order, with nuclei isolated shortly after repositioning. This time is designated as the TDP.

replication-timing program in vitro. We called this transition the TDP and demonstrated that this transition was coincident with the reorganization of chromatin to positions where it would reside for the rest of interphase (Fig. 2) (Dimitrova and Gilbert 1999). We identified a TDP for globally labeled early- and late-replication domains as well as specific loci comprising either facultative or constitutive heterochromatin in both mouse and hamster fibroblasts (Dimitrova and Gilbert 1999; Li et al. 2001; Wu et al. 2006; $\mathrm{Lu}$ et al. 2010). For example, the hamster $\beta$-globin locus acquired a late-replication-timing program coincident with its association with peripheral heterochromatin ( $\mathrm{Li}$ et al. 2001). The TDP takes place before the specification of replication origin sites (origin decision point, ODP), and several in vitro manipulations can disturb the selection of origins without affecting the replication-timing program (Dimitrova and Gilbert 1999; Lu et al. 2010), demonstrating that replication timing is established at the level of domains independent of origin usage.

Whereas our experiments were snapshots with fixed cell specimens, live cell imaging of specific chromosomal loci defined a very similar time after mitosis at which chromatin becomes anchored and movements constrained for the remainder of interphase (Leonhardt et al. 2000; Belmont 2001; Chubb et al. 2002; Walter et al. 2003). Moreover, studies in yeast have demonstrated that late replication and positioning to the nuclear periphery at a telomeric locus is established at some point during $\mathrm{G}_{1}$ phase (Raghuraman et al. 1997; Heun et al. 2001). Taken together, these experiments led us to propose a rather näive model (Gilbert 2001) showing that factors regulating replication timing may disperse during mitosis, and the anchorage of chromosome domains after mitosis could seed the self-assembly of spatially separated compartments of specific protein composition. These compartments would then set thresholds for initiation of replication, perhaps by regulating the accessibility of Sphase-promoting factors to the replication origins.

Since proposing this model, we have ruled out many processes and factors as candidates for regulators of replication timing but have not yet identified determinants of replication timing. Recently, we reasoned that in order for the timing determinant to be established in every $G_{1}$ phase, it must be lost at some point during the cell cycle; knowing when it is lost might help us to understand the nature of the determinant. Because such determinants were expected to be components of chromatin or 3D architecture, they should logically be lost during either S phase, when chromatin is reassembled, or mitosis, when the 3D architecture of the nucleus is reassembled. For this reason, we devised ways to induce a single round of reduplication within $\mathrm{G}_{2}$ phase nuclei by either washing $\mathrm{G}_{2}$-phase nuclei free of replication inhibitory factors and introducing them into Xenopus egg extracts or inhibiting cyclin-dependent kinase activities within living cells. In either case, replication initiated within $\mathrm{G}_{2}$-phase nuclei took place in a random temporal order ( $\mathrm{Lu}$ et al. 2010). We can conclude from this experiment that the replication-timing determinant(s) is assembled during early $\mathrm{G}_{1}$ phase and then lost during replication. This could occur by dilution of a chromatin mark at the replication fork, such as a protein or posttranslational modification. Although chromatin is assembled at the replication fork, there is evidence that some marks such as the centromeric histone variant (Jansen et al. 2007) and some histone modifications (Aoto et al. 2008; Scharf et al. 2009) are not restored until after mitosis. An alternative possibility is that the close proximity of two sister chromatids after replication may interfere with long-range chromatin interactions important for the replication-timing program $(\mathrm{Lu}$ et al. 2010).

In these same experiments ( $\mathrm{Lu}$ et al. 2010), we found that when cells exit the cell cycle and become quiescent, specific segments of chromatin can become substantially decondensed, yet when nuclei from quiescent cells were induced to replicate, replication followed the normal temporal sequence despite this chromatin reorganization. Together, these experiments suggest that chromosome architecture, as it is established at the TDP and visualized by conventional microscopy, is neither necessary nor sufficient to maintain replication timing. However, we cannot rule out architectural features that are not detected by microscopy. Moreover, 
these experiments do not rule out a role for 3D organization in the initial establishment of a replication-timing program. In fact, both the initial experiments identifying the TDP and compelling genome-wide analyses described below argue that chromosome architecture is intimately linked to replication timing.

\section{DEVELOPMENTAL CONTROL OF REPLICATION TIMING}

If replication timing is related to gene expression, one would expect it to be regulated during development. Although a relationship between replication timing and transcription has been observed for decades, until just a few years ago, there was virtually no direct evidence for dynamic changes in replication timing during development (Hiratani et al. 2004; Perry et al. 2004). A few dozen genes had been examined in different cell types with the finding that most genes replicate early in all cell types, whereas a few genes replicate early only in those cell types in which they are expressed (Gilbert 2002; Goren and Cedar 2003). However, these earlier studies were performed almost exclusively in established, usually transformed cell lines, so it was not possible to rule out whether differences in replication timing reflected genome rearrangements that may have accumulated in culture. The development of stem cell differentiation systems that undergo reasonably homogeneous transitions from one cell type to another have provided the first opportunity to query changes in the replication program; with the exception of random $\mathrm{X}$ inactivation, this was not previously possible (Keohane et al. 1996; Brockdorff 2002). In 2004, we and other investigators (Hiratani et al. 2004; Perry et al. 2004) surveyed replication timing of more than 50 genes during mouse embryonic stem cell (ESC) differentiation to neural precursors, identifying changes in replication timing for 13 genes residing within regions of high AT and long interspersed nuclear element sequence (LINE) content. This provided proof of principle for us to develop genome-wide methods to survey replication-timing changes in cultured cell differentiation models of early mammalian development, allowing us to identify the conditions that can elicit replication-timing change. Moreover, by comparing events during mouse and human ESC differentiation, we hoped to identify conserved aspects of the replication program that could suggest important functional relationships. We have since used this methodology to address some fundamental unanswered questions: To what extent is replication timing subject to developmental control? How are changes in replication timing related to changes in transcription/subnuclear organization? What features of regulation are conserved between human and mouse? Can we achieve a molecular definition of replication domains and replication foci?

To determine replication timing, cells are pulse-labeled with BrdU, stained for DNA content, and then sorted by fluorescence-activated cell sorting (FACS) into populations of cells in early or late $\mathrm{S}$ phase. The BrdU-substituted DNA synthesized during these two windows of S phase is immunoprecipitated with an anti-BrdU antibody, differentially labeled, and hybridized to a DNA array, and microarray data are plotted as a log ratio of early-to-late (E-to-L)-replicating DNA (Fig. 3). The data from such experiments immediately revealed easily visualized domains of differential replication timing, with well-defined boundaries that we could identify computationally by adapting an existing segmentation algorithm (Olshen et al. 2004; Venkatraman and Olshen 2007). Replication-timing profiles were nearly identical among different genetically polymorphic ESC lines for both human and mouse ESCs and their respective induced pluripotent stem cell lines (human-induced pluripotent-stem cells [hiP$\mathrm{SCs}$ ] and mouse-induced PSCs [miPSCs]) reprogrammed from differentiated cells back to the pluripotent state (Hiratani et al. 2008). However, when either mouse or human ESCs were differentiated to any of several different lineages, a global reorganization of replication domains occurred that affected approximately $20 \%$ of the genomes between any pair of cell types. Comparison of mouse and human replication-timing profiles revealed strong cell-type specific conservation. In addition, replication-timing changes resulted in a consolidation in the number of differentially replicating domains, with both early-replicating domains flanked by late domains consolidating into a single larger late domain (E-to-L consolidation) and late domains flanked by early domains consolidating into larger early domains (L-to-E consolidation).

Replication domains in any given mouse or human cell type could be quite variable in size - up to several megabases. However, in both species, the domains that changed replication timing were uniform in size, on the order of 400 $800 \mathrm{~kb}$, providing evidence for a minimal basic unit of replication-timing change. Interestingly, this size conforms closely to cytogenetic estimates of the amount of DNA in each of the many replication foci seen when cells are pulselabeled with nucleotide analogs (Ma et al. 1998). Together, these data provide the best evidence to date for the existence of molecular units of replication timing that may be the molecular equivalent to cytogenetically defined replication foci. Larger replication domains likely consist of several of these smaller units whose individual replication times cannot be distinguished. In fact, cytogenetic studies have identified many regions throughout the genome in which adjacent replication foci are sequentially activated within $1 \mathrm{~h}$ of one another (Sadoni et al. 2004; Maya-Mendoza et al. 2010).

\section{REPLICATION TIMING AND TRANSCRIPTION: PROMOTER-SPECIFIC RELATIONSHIP}

By performing transcription microarray analyses in many lines of the same cell type, we demonstrated that there is a strong positive correlation between early replication and transcription, as expected from prior studies in all cell types and species except for yeasts (Gilbert 2010). Because we had genome-wide data at high resolution, unlike the earlier studies, we were able to conclude that approximately $75 \%$ of genes are replicated in the first half of S phase, with mostly nongenic DNA replicating in late $S$ in all cell types examined to date (Hiratani et al. 2008; T Ryba, unpubl.). During differentiation, up-regulated and down-regulated genes that also change replication timing are strongly enriched for L-to-E and E-to-L changes, respectively. How- 
A

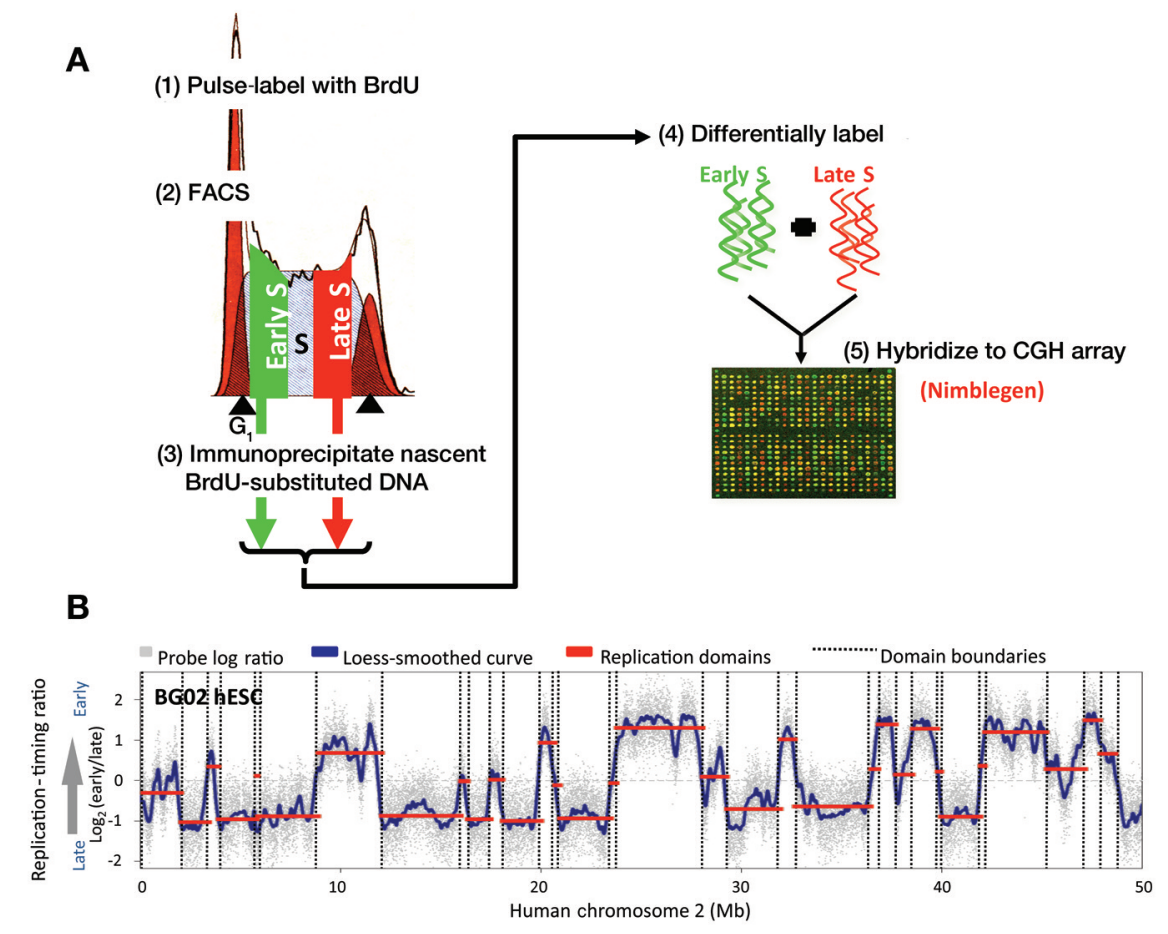

B

Figure 3. Genome-wide replication-timing analysis. (A) Cells are pulse-labeled with BrdU and then are sorted into early and late stages of S phase by flow cytometry. The BrdU-substituted DNA synthesized during these two windows of S phase is immunoprecipitated with an anti-BrdU antibody, differentially labeled, and hybridized to a DNA array. (B) Replication-timing profile of BG02 hESC. Probes (gray dots) are plotted as $\log$ ratios $\left(=\log _{2}\right.$ [early/late]) along chromosome 2 . A local polynomial smoothing (loess) curve is overlaid (blue). Also overlaid are replication domains (red lines) and their boundaries (dotted lines) identified by segmentation. ( $A$, Adapted from Hiratani et al. 2008; B, adapted, with permission, from Ryba et al. 2010.)

ever, the relationship is clearly not direct; many genes change transcription with no change in replication time and vice versa. Moreover, late-replicating expressed genes and even a rare class of genes are up-regulated on a switch from E-to-L replication. Most of these exceptions to the rule were genes with CpG-rich promoters that, when examined in isolation, showed no relationship at all to replication timing. Hence, the relationship between transcription and replication timing is promoter specific. One caveat to all transcription comparisons so far is that because they use microarray analyses of well-characterized genes, they do not take noncoding transcription into account. Because the amount of noncoding transcription that takes place in the genome is still controversial (van Bakel et al. 2010), there could still be relationships that we are missing. For example, it is possible that replication timing could be a reflection of the average transcriptional activity (coding and noncoding) across large chromosomal domains, as has been suggested in Drosophila cells based on the relationship between replication timing and the density of RNA polymerase II within large chromosome domains (MacAlpine et al. 2004).

An important long-standing question in the field asks which change occurs first: replication timing or transcription. This was difficult to answer. As discussed above, there is a preference for genes in switching domains to change coordinately with replication timing. Moreover, the E-to-L changes in genes that are mostly down-regulated precede the loss of pluripotency, whereas L-to-E changes in genes that are mostly activated in a lineage-specific fashion follow loss of pluripotency, as if the cell must first move pluripotency-associated genes to a late-replicating environment before moving lineage-specific genes to an early-replicating compartment. However, a simple temporal order could not be assigned among changes in replication timing and transcription; either change could come first. Of course, ensemble genomics methods cannot identify whether any of these events are causal at individual gene loci; we can only say that there is no universal rule governing the relationship between replication timing and transcription.

\section{REPLICATION TIMING AND HISTONE MODIFICATIONS: A COMPLEX RELATIONSHIP}

The availability of replication-timing maps for many different cell types also allowed us to relate replication timing of each domain to the density of several epigenetic marks in mouse ESCs and neuronal precursor cells (NPCs) as well as in human lymphoblasts (Hiratani et al. 2008; Ryba et al. 2010). As expected, we found a positive correlation in all cell types between early replication and active chromatin marks (e.g., H3K4me1,me2,me3; H3K9ac; H3K36me3) that was similar to the association with transcription. However, we were unable to find a similarly strong negative correlation to repressive chromatin marks $\mathrm{H} 3 \mathrm{~K} 9 \mathrm{me} 3$ or 
H3K27me3. Because there are only a few genome-wide data sets for histone marks, we still cannot rule out celltype variation in these correlations or the possibility that a lack of correlation results from poor antibody specificity. In fact, using new monoclonal antibodies raised against $\mathrm{H} 3 \mathrm{~K} 9 \mathrm{me} 3$ and $\mathrm{H} 3 \mathrm{~K} 27 \mathrm{me} 3$, we have observed a weak but significant correlation between these marks and late replication in IMR90 primary human fibroblasts (T Chandra et al., unpubl.). We did find evidence for an epigenetic signature bordering early-replicating domains enriched in histone marks indicative of active transcription, including H3K36me3 and depleted repressive marks. The position of this signature was different within each individual earlyreplicating domain but was typically within $100 \mathrm{~kb}$ of the domain boundary. The significance of this finding remains to be determined, but because the domain boundaries are likely to be regions of suppressed origin activity where replication forks are forced to travel unidirectionally until they encounter a later-replicating domain (Guan et al. 2009), it is possible that active transcription can function to counteract the spreading of some chromatin feature that prevents origin firing, thus establishing a boundary for permissive origin activity leading to early replication.

Intriguingly, $\mathrm{H} 3 \mathrm{~K} 9 \mathrm{me} 2$ was strongly correlated to late replication in both human and mouse cell lines and was found by immunofluorescence to be enriched at the nuclear periphery (Yokochi et al. 2009; Ryba et al. 2010). Genetic knockout of the histone methyltransferase G9a eliminated all detectable $\mathrm{H} 3 \mathrm{~K} 9 \mathrm{me} 2$ at the nuclear periphery and resulted in the activation of 167 genes that were localized to the nuclear periphery. However, G9a loss had no detectable effect on late-replicating and genome-wide timing or localization of several candidate genes, demonstrating that $\mathrm{H} 3 \mathrm{~K} 9 \mathrm{me} 2$ is associated with, but not necessary for, late replication or peripheral localization. This is consistent with myriad anecdotal studies of replication timing in cells harboring disruptions in various genes; effects of such knockouts are either minimal or highly localized (Wu et al. 2005, 2006; Jørgensen et al. 2007). In any case, G9a appears to be sufficient to maintain repression of a set of genes that are within the late-replicating peripheral compartment of the nucleus. Why these up-regulated genes are localized to this compartment and whether the compartment participates in the mechanism(s) that repress these genes remain to be determined.

Overall, our results suggest that there is no one-to-one relationship to chromatin marks and that replication timing is quite resilient to mutations in individual chromatin proteins. This could be due to redundancy if a combination of many chromatin features determines replication timing, or it could be that the determinant(s) simply has not been discovered. If replication timing reflects or represents higherorder organization of chromatin, it should come as no surprise that it results from complex features of chromatin. Moreover, replication domains are not binary early versus late replicating but instead replicate at many different specific times throughout the course of S phase. Hence, it is possible that complex combinations of chromatin proteins and histone marks give rise to subtle differences in replication timing. It is also possible that as yet undiscovered cis-acting sites modulate replication timing and by averaging the density of histone marks across domains, as we have done, we are overlooking important individual elements. Another hypothesis is that it is only the chromatin marks at replication origins that are important for replication timing, and there do appear to be some local chromatin effects at certain origins (Goren et al. 2008; Zhou et al. 2010). However, because timing is determined independent of origins (see above), and each individual cell is initiating from a different subset of origins (Gilbert 2010), and simultaneously firing origins within the same domain can harbor very different histone modifications (Prioleau et al. 2003), this seems to be an oversimplification.

\section{GENOME-WIDE STUDIES LINK REPLICATION TIMING TO 3D CHROMATIN ARCHITECTURE}

The most striking finding from all of these comparisons between replication timing and other epigenetic marks was a nearly perfect correlation to the frequency of chromatin interactions when replication timing within human B lymphocytes was compared with high-resolution chromatin conformation capture (Hi-C) maps in the same cell type. Hi-C maps provide a measure of the relative frequency with which sequences throughout the genome interact within a population of cells. Living cells are fixed to preserve 3D organization, and DNA within fixed chromatin is cut with a restriction enzyme and religated to covalently link DNA segments that were in close proximity. All of these junctions are then sequenced to reveal the linkages (Lieberman-Aiden et al. 2009). Results from Hi-C mapping have led to the conclusion that chromatin interactions are spatially segregated into two nuclear compartments designated $\mathrm{A}$ and $\mathrm{B}$, such that sequences in compartment A can interact with other sequences in the same compartment but not with sequences in compartment $B$ and vice versa. When these data were plotted linearly as an eigenvector, compartments $\mathrm{A}$ and $\mathrm{B}$, including localized variations in interaction frequencies within the compartments, corresponded remarkably to early- and late-replicating domains (Ryba et al. 2010). In fact, compartment B had a higher density of interactions, consistent with generally more compact late-replicating chromatin. This is the highest correlation that we have identified for any functional or structural chromosomal property. The correlation was found on every chromosome and on some chromosomes was as high as data that we obtain in replicate experiments!

This result provides evidence from completely different methodologies that different replication domains are spatially separated in the nucleus. Figure 4 compares the model developed by Lieberman-Aiden et al. (2009), in which each interacting domain self-interacts to form what they call a fractal globule. Chromatin within each globule interacts most frequently with sequences within the same globule, can also interact with other globules within the same compartment, but cannot interact with chromatin in the alternate compartment. This looks remarkably like our model, proposed $\sim 10$ years earlier, to describe spatially separated early- and late-replicating domains (Gilbert 2001). Together, these data strongly support the notion that chromatin 

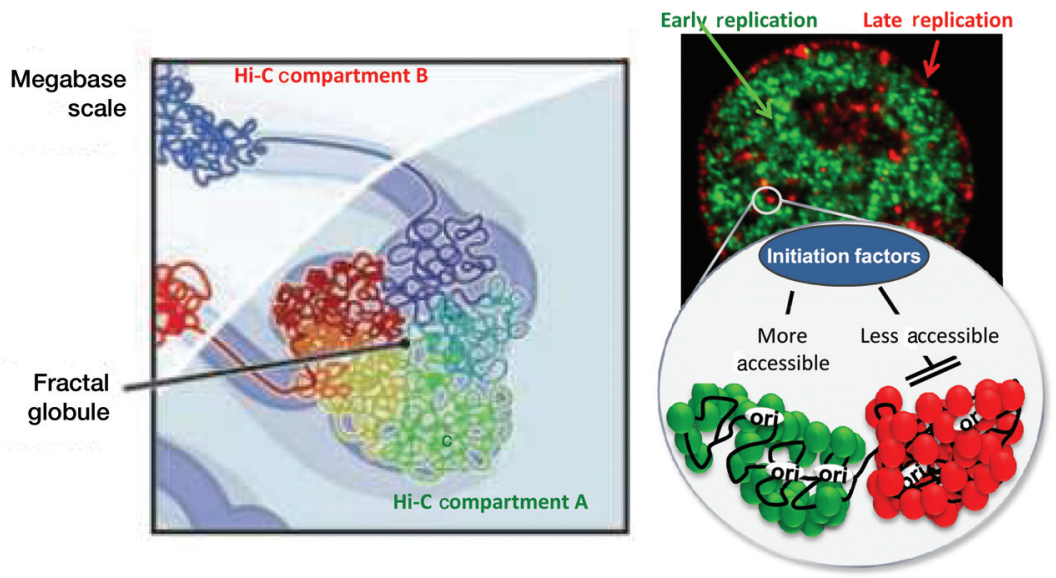

Figure 4. Genome-wide studies link replication timing to 3D chromatin architecture. $(A)$ In the model developed by Lieberman-Aiden et al. (2009), based on a recent genome-wide analysis of chromatin interactions, chromosomes consist of a series of fractal globules defined by these interactions. Chromatin can interact with other globules within the same compartment but cannot interact with chromatin in the alternate compartment. $(B)$ Replication foci labeled either early (green) or late (red) during S phase are depicted as fractal globules in separate nuclear compartments. Hi-C compartment B has a higher density of chromatin interactions, indicative of more compact chromatin and corresponding to late-replicating domains, depicted here as rendering replication origins less accessible to initiation factors. ( $A$, Adapted, with permission, from Lieberman-Aiden et al. 2009 [AAAS]; B, adapted, with permission, from Ryba et al. 2010.)

in close proximity replicates at similar times and stays together as a distinct chromosomal unit of organization that can be visualized cytogenetically as foci, by replication-timing profiling as replication domains, or by $\mathrm{Hi}-\mathrm{C}$ as regions of self-interaction.

\section{REPLICATION-TIMING PROFILES REVEAL CLEAR EPIGENETIC DISTINCTIONS AMONG CLOSELY RELATED CELL TYPES}

We have profiled a large number of cell types (see http:// www.replicationdomain.org), and if we divide the genome into $11,000200-\mathrm{kb}$ segments, we can use hierarchical clustering to reveal the relatedness among various cell types with respect to their replication-timing programs. In Figure 5 , the profiles of 22 cell lines are divided into seven cell states. Overall, we found that close to half of the genome exhibits changes in replication timing at some stage of development. Immediately obvious is that all embryonic cell types cluster together, whereas more differentiated cell types cluster, providing that they are from the same germ layer. Surprisingly, cell types corresponding to early epiblast (i.e., early-primitive ectoderm-like [EPL] and EBM3/differentiation day 3) and late epiblast (i.e., epiblast-derived stem cells) that represent early differentiation intermediates separated by only one or two cell cycles, form widely separated clusters. Late epiblast clusters more closely with early committed cell types and very closely with a definitive ectoderm-like cell population (differentiation day 6) and NPCs (differentiation day 9). These two cell types are almost identical in transcriptional profile (including the expression of transcription factors regulating pluripotency), both make teratomas, and neither can make chimeric mice. Despite these similarities, EPL and EBM3 are fully reversible back to the ESC state with a simple media change, whereas EpiSCs are not. In fact, the efficiency of iPS formation with EpiSCs is similar to that of adult cells (Bao et al. 2009; Guo et al. 2009). Hence, they have very different phenotypes with respect to reprogramming, and they have very different replication profiles, despite similar transcription profiles. These results illustrate the ability of replication-timing profiling to identify important epigenetic distinctions between closely related cell types.

The replication-timing changes among these cell types are mostly within regions containing genes that are switching from early to late and are then remaining late replicating throughout the rest of development, consistent with functionally significant changes in pluripotency-specific genes. This is reminiscent of $\mathrm{X}$ inactivation in that the switch to late replication and irreversible silencing that persists clonally throughout development occurs in this same stage of development and suggests that we may have identified an autosomal equivalent of facultative heterochromatinization (Hiratani and Gilbert 2010).

\section{LATE REPLICATION AS A BARRIER TO REPROGRAMMING}

In the relatively inefficient process of deriving iPSCs, many colonies arise that share the morphology of iPSCs but fail to express several pluripotency genes. An interesting question is whether these partially induced pluripotent stem cells (piPSCs) have become stabilized at arbitrary stages in the reprogramming process or whether they are all encountering a common roadblock important for the final stages of reprogramming. Because replication profiles can distinguish closely related cell types, we profiled three mouse embryonic fibroblast (MEF)-derived piPSC clones harboring independent reprogramming vector insertion sites (Hiratani et al. 2010). As seen in Figure 5, piPSCs 


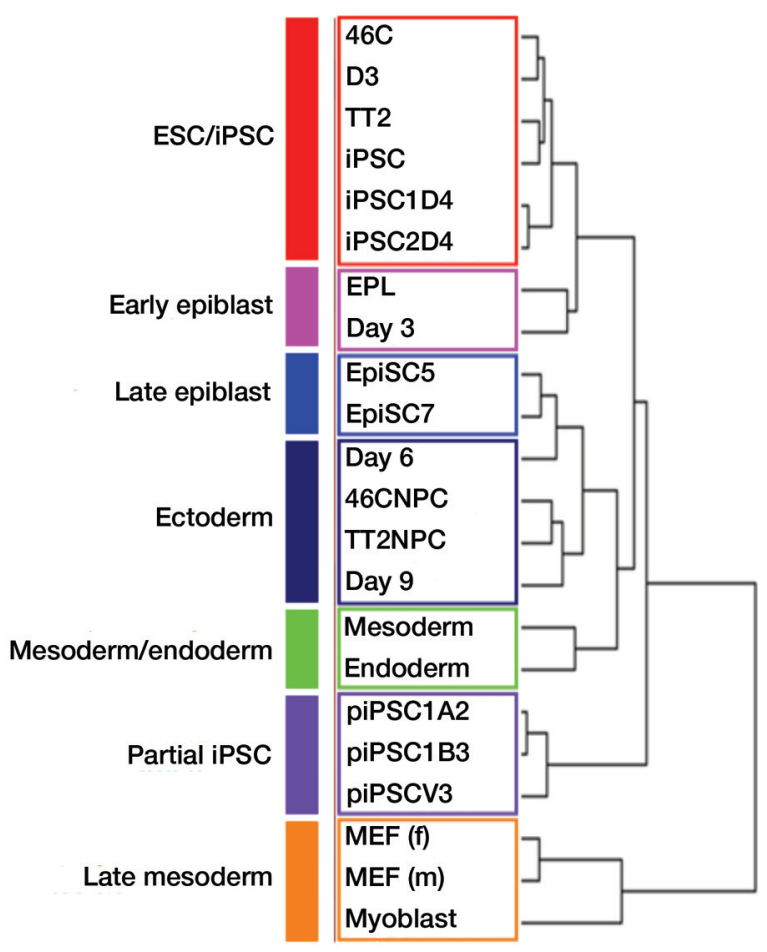

Figure 5. Dendrogram of replication-timing changes during mouse embryogenesis. Twenty-two cell lines representing seven stages (differently colored) of mouse embryogenesis were profiled for replication timing as described in Figure 3. The genome was then divided into 11,000 200-kb segments, hierarchical clustering of the replication times of each of these segments for each cell line was performed, and the data were displayed as a dendrogram. Names of cell lines and differentiation states can be found in Hiratani et al. (2010). (Adapted, with permission, from Hiratani et al. 2010.)

form their own independent cluster, nonetheless more related to early cell types than the downstream MEF and myoblast lineages. In fact, all three piPSCs had virtually identical overall profiles (genome-wide Pearson's correlation $R^{2}=0.94$ ), suggesting that they were indeed all arrested at a common epigenetic barrier. Their profiles were distinct from fully reprogrammed iPSCs $\left(R^{2}=0.77\right)$ in that the inactive $\mathrm{X}$ chromosome remained late replicating, and differences were observed in very specific regions containing genes such as the pluripotency-specific gene Rexl (Fig. $6 \mathrm{~A}, \mathrm{~B})$, in which there is a complete lack of reprogramming of replication timing across an $\sim 1$-Mb region.

What is happening to the expression of the genes within these regions where replication timing has not been reprogrammed to the pluripotent state? In Figure 6C, those chromosomal segments that switch significantly to later replication in epiblast stages and remain late during the course of development are clustered together in a heat map representing the replication timing in each of the seven cell states indicated in Figure 5. To the right of the heat map are the correlations of PSC and piPSC to ESC transcript levels, assayed by transcription microarray. Genes within this group of segments are particularly resistant to reprogramming, showing a very low correlation $\left(R^{2}=\right.$ 0.24 ) of piPSC to ESC transcript levels relative to either these same gene transcript levels in fully reprogrammed iPSCs $\left(R^{2}=0.90\right)$ or to the global transcriptional correlation, which is high for both iPSCs and piPSCs. Together, these results strongly suggest that late replication is associated with the heritable silencing of pluripotency genes during the epiblast stages, comprising an epigenetic impediment to reprogramming. This same analysis revealed replication domains that are early replicating in MEFs and difficult to reprogram back to the late-replicating silenced state in these piPSCs. These domains contained many MEF-specific genes. This suggests the interesting possibility that iPSCs generated from different tissues encounter unique cell-type-specific barriers.

\section{BIOLOGICAL SIGNIFICANCE OF NUCLEAR COMPARTMENTS}

Our model (Figs. 2 and 4) predicts that large replicationtiming switches should be accompanied by changes in spatial compartmentalization. In fact, when we perform fluorescence in situ hybridization (FISH) on several genes within the "difficult to reprogram" regions shown in Figure $6 \mathrm{C}$, we find that these genes move from the interior to the periphery during this critical epiblast transition (Hiratani et al. 2010). Interestingly, neural-specific genes that switched from L-to-E replication in a neural differentiation protocol also moved from the periphery to the nuclear interior. As a method, FISH is laborious and difficult when analyzing more than a handful of loci. However, we think that this spatial reorganization during differentiation is quite extensive because an analysis of total chromatin by electron spectroscopic imaging (ESI) revealed a dramatic compaction of chromatin at the periphery of the nucleus that takes place during this same early developmental period (Hiratani et al. 2010). It is possible that compaction may accompany replication domain temporal consolidation and movement toward the periphery. Indeed, when we examined the distances between several FISH probes across approximately $5 \mathrm{Mb}$ containing an E-to-L consolidating domain, movement toward the periphery was accompanied by a dramatic spatial compaction of this locus. Moreover, when we examined the distances between pairs of probes spaced across this same region, a linear relationship between spatial distance and base-pair distance was observed for all pairs of probes except those within the E-to-L switching domain while in its early-replicating configuration (S Takebayashi, unpubl.). This result suggests the existence of a mechanism to decompact and move this domain into a different subnuclear compartment specifically in pluripotent cells. We have not yet examined L-toE replication-timing consolidation for effects on chromatin compaction.

A time-course analysis of transcription, replication timing, and $3 \mathrm{D}$ genome organization has revealed that, whereas transcription and replication timing change gradually and do not necessarily follow a specific temporal sequence, 3D organizational changes in subnuclear compartmentalization, particularly proximity to the nuclear periphery, generally occur in a more abrupt fashion (Hiratani et al. 2010). Several additional observations are consistent with this no- 


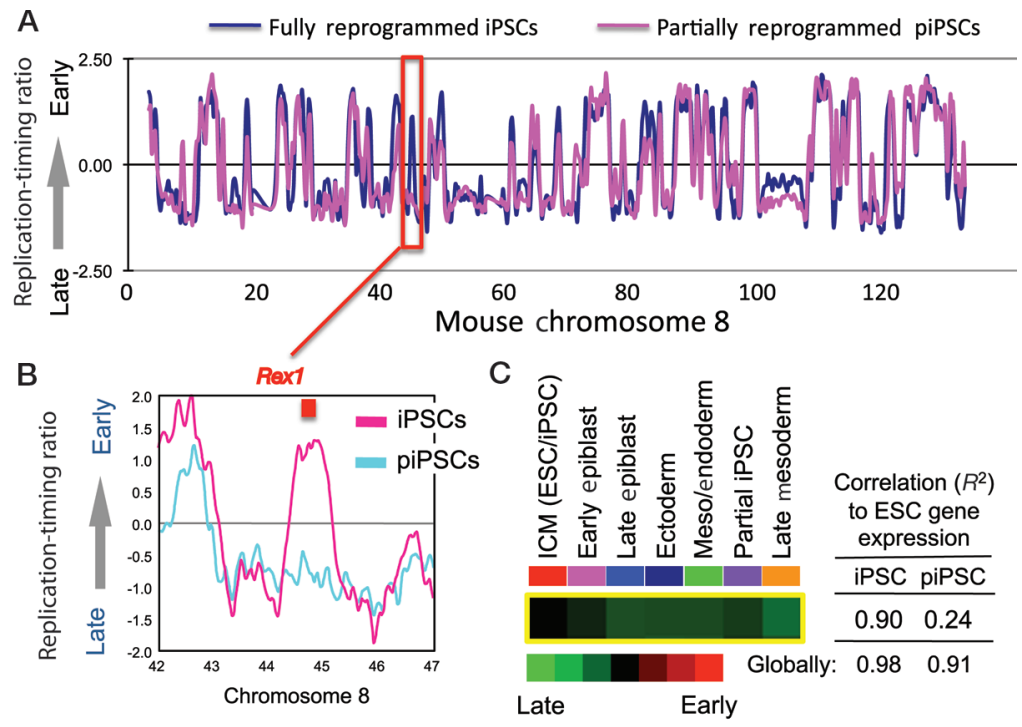

Figure 6. Replication timing as a barrier to reprogramming. (A) Replication-timing profiles for mouse iPSCs (blue) and piPSCs (pink) are overlaid. Microarray data are plotted as logarithmic ratios $\left(=\log _{2}\right.$ [early/late]) along mouse chromosome 8 , and a local polynomial smoothing (loess) curve is applied, as in Figure 3. (B) Expansion of a 5-Mb region containing the Zfp42/RexI gene reveals an 700$\mathrm{kb}$ replication domain that has been reprogrammed back to the early-replicating state in iPSCs but is completely late replicating in piPSCs. (C) Heat map of replication timing in the indicated cell types for those replication domains that switch significantly from Eto-L replication during development. To the right of the heat map are Pearson's $R^{2}$ values comparing transcriptional microarray data for the genes found within this group of domains between iPSCs or piPSCs and ESCs. The much lower piPSC correlation value $\left(R^{2}\right.$ $=0.24$ ) suggests that genes within these late-replicating domains are particularly resistant to transcriptional reprogramming. However, comparison of transcriptional microarray data for all annotated genes (globally) shows that overall transcription is generally well reprogrammed in piPSCs; only those genes within replication-timing switch regions are difficult to reprogram.

tion. First, when we look at the percentage of expressed genes replicated at different time intervals during $\mathrm{S}$ phase, in any mouse or human cell type, genes replicated throughout the first half of $\mathrm{S}$ phase $(75 \%$ of genes in any given cell line) show no correlation between the probability of expression and replication timing. However, for the remaining genes replicated in the second half of S phase, there is a very strong relationship between replication time and probability of expression (Hiratani et al. 2008, 2009; Ryba et al. 2010). This binary relationship suggests that it is only those replication-timing changes taking place during the second half of S phase that have consequences for transcription.

What could be so special about this interval? Recall that the entire first half of S phase is devoted to the replication of chromatin distributed throughout the interior of the nucleus; it is nearly impossible to distinguish the spatial patterns of replication within cells at different early S-phase time periods. This is followed by a dramatic transition to peripheral replication in the middle of S phase, which leads us to speculate that it is only that class of replication-timing changes associated with subnuclear position changes that are relevant to transcription. This also suggests that, in principle, we should be able to predict genes that undergo spatial position changes during differentiation based on whether they move through this replicationtime interval. And, in fact, if we look at a given locus during the differentiation time interval when subnuclear position is changing, the replication timing of that locus is found to be moving through the mid S-phase time interval (Hiratani and Gilbert 2009; Hiratani et al. 2010).
Together with the results discussed above regarding transcriptional control of different classes of genes, it appears that the relationship between replication timing and transcription is restricted to a specific class of promoters (e.g., $\mathrm{CpG}$ poor) and is only functionally relevant when replication timing changes through the middle of $\mathrm{S}$ phase, when the replication-timing change is predictive of a change in subnuclear compartment.

\section{CONCLUSIONS}

Our genome-wide studies during the last several years have substantially clarified the relationship between replication timing and transcriptional output, teasing apart the complexity of this relationship and defining a set of conditions required for associations to have functional significance. At least $50 \%$ of the genome is subject to developmental control of replicating timing, coordinated with changes in transcription. However, there are clearly many genes whose transcription is not affected by replication timing. The promoters and regulatory elements of such genes may have strong nucleosome-positioning sequences, $\mathrm{CpG}$ islands, or otherwise dominating local sequence information that override any influence of the factors related to replication timing. However, for those genes that are affected, replication timing is indicative of a somatically heritable epigenetic state that is difficult to reverse. Moreover, no genes appear to be affected if a change in replication timing is restricted to the first half of $\mathrm{S}$ phase. There is less genomewide data localizing chromatin proteins and histone marks 
than steady-state transcript measurements, but so far, early replication aligns with marks indicative of active transcription as expected, whereas repressive chromatin marks show a complex relationship that will require further experimentation to understand.

Replication timing is also clearly related to subnuclear position, as predicted from earlier studies, but examination of dynamic changes in these properties reveals that the relationship is far from one to one. Our results suggest that only those replication-timing changes traversing the middle of S phase are related to subnuclear position and that a transition in replication timing through this period of $\mathrm{S}$ phase is predictive of a subnuclear position change. This implies that genes whose transcription is unaffected by such replication-timing changes are also unaffected by subnuclear position (Luo et al. 2009). A further complexity is that subnuclear position changes are probabilistic, and it is not clear that a late-replicating domain would be localized at the periphery or nucleolar periphery in every cell in a population. At present, it is also difficult to evaluate the degree to which replication timing is heterogeneous from cell to cell. Finally, the close alignment between Hi-C and replication-timing maps suggests that it is proximity and local 3D architecture rather than subnuclear position per se that is an important predictor of replication timing. Altogether, it is clear that the DNA that resides close together in the nucleus replicates as a coordinately regulated unit, punctuated by intervening DNA that is suppressed for initiation activity and traverses time and space via a unidirectional replication fork.

When will we get to cause and effect? It should be amply clear now from myriad studies of chromatin mechanisms that "chicken or the egg" questions are particularly difficult to address because chromatin mechanisms work by positively reinforcing feedback loops that muddy the definitions of cause and effect. In the life cycle of a cell, 3D architecture is assembled after mitosis at the TDP, likely influenced by a combination of memory elements linked to mitotic chromatin (Smale and Fisher 2002) and the constellation of chromatin-binding factors available at this time, controlled by signals in the environment and the symmetry of cell division (Egli et al. 2008). Because the decision to proliferate or enter quiescence has not been made at this time, it is correct to say that the replication-timing program reflects the $3 \mathrm{D}$ architecture rather than vice versa and is likely dictated in some unknown way by this architecture. However, once entering $\mathrm{S}$ phase, the timing program dictates spatiotemporal segregation in the assembly of chromatin, and it is reasonable to assume that this influences the type of chromatin assembled across entire 400$800-\mathrm{kb}$ domains. It also seems likely that aspects of this chromatin structure are maintained through mitosis to influence the 3D architecture assembled in the next cell cycle. If this is true, it is also correct to say that 3D architecture reflects replication timing. Dissecting the mechanisms linking these two complex features of chromosomes is not going to be easy, and evaluating the significance of these features for functional activities of chromosomes will require identification of the mechanisms linking them. For now, we can say that, regardless of mechanism or signifi- cance, replication timing is an easily measurable property of chromosomes that is directly predictive of, if not directly linked to, complex higher-order features of chromosome architecture.

\section{REFERENCES}

Aoto T, Saitoh N, Sakamoto Y, Watanabe S, Nakao M. 2008. Polycomb group protein-associated chromatin is reproduced in postmitotic $\mathrm{G}_{1}$ phase and is required for $\mathrm{S}$ phase progression. J Biol Chem 283: 18905-18915.

Bao S, Tang F, Li X, Hayashi K, Gillich A, Lao K, Surani MA. 2009. Epigenetic reversion of post-implantation epiblast to pluripotent embryonic stem cells. Nature 461: 1292-1295.

Belmont AS. 2001. Visualizing chromosome dynamics with GFP. Trends Cell Biol 11: 250-257.

Berezney R, Dubey DD, Huberman JA. 2000. Heterogeneity of eukaryotic replicons, replicon clusters, and replication foci. Chromosoma 108: 471-484.

Bickmore WA, Carothers AD. 1995. Factors affecting the timing and imprinting of replication on a mammalian chromosome. $J$ Cell Sci 108: 2801-2809.

Brockdorff N. 2002. X-chromosome inactivation: Closing in on proteins that bind Xist RNA. Trends Genet 18: 352-358.

Chubb JR, Boyle S, Perry P, Bickmore WA. 2002. Chromatin motion is constrained by association with nuclear compartments in human cells. Curr Biol 12: 439-445.

Dimitrova DS, Gilbert DM. 1999. The spatial position and replication timing of chromosomal domains are both established in early G1 phase. Mol Cell 4: 983-993.

Egli D, Birkhoff G, Eggan K. 2008. Mediators of reprogramming: Transcription factors and transitions through mitosis. Nat Rev Mol Cell Biol 9: 505-516.

Gilbert DM. 2001. Nuclear position leaves its mark on replication timing. J Cell Biol 152: F11-F16.

Gilbert DM. 2002. Replication timing and transcriptional control: Beyond cause and effect. Curr Opin Cell Biol 14: 377-383.

Gilbert DM. 2010. Evaluating genome-scale approaches to eukaryotic DNA replication. Nat Rev Genet 11: 673-684.

Gilbert DM, Gasser SM. 2006. DNA replication and nuclear architecture. DNA replication and human disease (ed. ML DePamphilis), pp. 175-196. Cold Spring Harbor Laboratory Press, Cold Spring Harbor, NY.

Goren A, Cedar H. 2003. Replicating by the clock. Nat Rev Mol Cell Biol 4: 25-32.

Goren A, Tabib A, Hecht M, Cedar H. 2008. DNA replication timing of the human $\beta$-globin domain is controlled by histone modification at the origin. Genes Dev 22: 1319-1324.

Guan Z, Hughes CM, Kosiyatrakul S, Norio P, Sen R, Fiering S, Allis CD, Bouhassira EE, Schildkraut CL. 2009. Decreased replication origin activity in temporal transition regions. J Cell Biol 187: 623-635.

Guo G, Yang J, Nichols J, Hall JS, Eyres I, Mansfield W, Smith A. 2009. Klf4 reverts developmentally programmed restriction of ground state pluripotency. Development 136: 1063-1069.

Hayashi MT, Takahashi TS, Nakagawa T, Nakayama J, Masukata H. 2009. The heterochromatin protein Swi6/HP1 activates replication origins at the pericentromeric region and silent matingtype locus. Nat Cell Biol 11: 357-362.

Heun P, Laroche T, Raghuraman MK, Gasser SM. 2001. The positioning and dynamics of origins of replication in the budding yeast nucleus. J Cell Biol 152: 385-400.

Hiratani I, Gilbert DM. 2009. Replication timing as an epigenetic mark. Epigenetics 4: 93-97.

Hiratani I, Gilbert DM. 2010. Autosomal lyonization of replication domains during early mammalian development. In The cell biology of stem cells (ed. E Meshorer and K Plath). Landes Bioscience/Madame Curie Bioscience Database.

Hiratani I, Leskovar A, Gilbert DM. 2004. Differentiation-induced replication-timing changes are restricted to AT-rich/long interspersed nuclear element (LINE)-rich isochores. Proc Natl Acad Sci 101: 16861-16866. 
Hiratani I, Ryba T, Itoh M, Yokochi T, Schwaiger M, Chang CW, Lyou Y, Townes TM, Schübeler D, Gilbert DM. 2008. Global reorganization of replication domains during embryonic stem cell differentiation. PLoS Biol 6: 1544-9173.

Hiratani I, Takebayashi S, Lu J, Gilbert DM. 2009. Replication timing and transcriptional control: Beyond cause and effect (part II). Curr Opin Genet Dev 19: 142-149.

Hiratani I, Ryba T, Itoh M, Rathjen J, Kulik M, Papp B, Fussner E, Bazett-Jones DP, Plath K, Dalton S, et al. 2010. Genomewide dynamics of replication timing revealed by in vitro models of mouse embryogenesis. Genome Res 20: 155-169.

Huberman JA. 1991. Cell cycle control of initiation of eukaryotic DNA replication. Chromosoma 100: 419-423.

Jackson DA, Pombo A. 1998. Replicon clusters are stable units of chromosome structure: Evidence that nuclear organization contributes to the efficient activation and propagation of $\mathrm{S}$ phase in human cells. J Cell Biol 140: 1285-1295.

Jansen LE, Black BE, Foltz DR, Cleveland DW. 2007. Propagation of centromeric chromatin requires exit from mitosis. J Cell Biol 176: 795-805.

Jørgensen HF, Azuara V, Amoils S, Spivakov M, Terry A, Nesterova T, Cobb BS, Ramsahoye B, Merkenschlager M, Fisher AG. 2007. The impact of chromatin modifiers on the timing of locus replication in mouse embryonic stem cells. Genome Biol 8: R169.

Keohane AM, O’Neill LP, Belyaev ND, Lavender JS, Turner BM. 1996. X-Inactivation and histone H4 acetylation in embryonic stem cells. Dev Biol 180: 618-630.

Lande-Diner L, Zhang J, Cedar H. 2009. Shifts in replication timing actively affect histone acetylation during nucleosome reassembly. Mol Cell 34: 767-774.

Leonhardt H, Rahn HP, Weinzierl P, Sporbert A, Cremer T, Zink D, Cardoso MC. 2000. Dynamics of DNA replication factories in living cells. J Cell Biol 149: 271-280.

Li F, Chen J, Izumi M, Butler MC, Keezer SM, Gilbert DM. 2001. The replication timing program of the Chinese hamster $\beta$-globin locus is established coincident with its repositioning near peripheral heterochromatin in early G1 phase. J Cell Biol 154: 283-292.

Lieberman-Aiden E, van Berkum NL, Williams L, Imakaev M, Ragoczy T, Telling A, Amit I, Lajoie BR, Sabo PJ, Dorschner MO, et al. 2009. Comprehensive mapping of long-range interactions reveals folding principles of the human genome. Science 326: 289-293.

Lu J, Li F, Murphy CS, Davidson MW, Gilbert DM. 2010. G2 phase chromatin lacks determinants of replication timing. J Cell Biol 189: 967-980.

Luo L, Gassman KL, Petell LM, Wilson CL, Bewersdorf J, Shopland LS. 2009. The nuclear periphery of embryonic stem cells is a transcriptionally permissive and repressive compartment. $J$ Cell Sci 122: 3729-3737.

Ma H, Samarabandu J, Devdhar RS, Acharya R, Cheng P, Meng C, Berezney R. 1998. Spatial and temporal dynamics of DNA replication sites in mammalian cells. J Cell Biol 143: 1415-1425.

MacAlpine DM, Rodriguez HK, Bell SP. 2004. Coordination of replication and transcription along a Drosophila chromosome. Genes Dev 18: 3094-3105.

Manders EM, Kimura H, Cook PR. 1999. Direct imaging of DNA in living cells reveals the dynamics of chromosome formation. J Cell Biol 144: 813-821.

Maya-Mendoza A, Olivares-Chauvet P, Shaw A, Jackson DA. 2010. S phase progression in human cells is dictated by the genetic continuity of DNA foci. PLoS Genet 6: e1000900.

Nakamura H, Morita T, Sato C. 1986. Structural organization of replicon domains during DNA synthetic phase in the mammalian nucleus. Exp Cell Res 165: 291-297.

Nakayasu H, Berezney R. 1989. Mapping replicational sites in the eucaryotic cell nucleus. J Cell Biol 108: 1-11.

O'Keefe RT, Henderson SC, Spector DL. 1992. Dynamic organization of DNA replication in mammalian cell nuclei: Spatially and temporally defined replication of chromosome-specific $\alpha$ satellite DNA sequences. J Cell Biol 116: 1095-1110.

Olshen AB, Venkatraman ES, Lucito R, Wigler M. 2004. Circular binary segmentation for the analysis of array-based DNA copy number data. Biostatistics 5: 557-572.

Panning MM, Gilbert DM. 2005. Spatio-temporal organization of DNA replication in murine embryonic stem, primary, and immortalized cells. J Cell Biochem 95: 74-82.

Perry P, Sauer S, Billon N, Richardson WD, Spivakov M, Warnes G, Livesey FJ, Merkenschlager M, Fisher AG, Azuara V. 2004. A dynamic switch in the replication timing of key regulator genes in embryonic stem cells upon neural induction. Cell Cycle 3: $1645-1650$.

Pope BD, Hiratani I, Gilbert DM. 2010. Domain-wide regulation of DNA replication timing during mammalian development. Chromosome Res 18: 127-136.

Prioleau MN, Gendron MC, Hyrien O. 2003. Replication of the chicken $\beta$-globin locus: Early-firing origins at the $5^{\prime}$ HS4 insulator and the $\rho$ - and $\beta^{\mathrm{A}}$-globin genes show opposite epigenetic modifications. Mol Cell Biol 23: 3536-3549.

Raghuraman M, Brewer B, Fangman W. 1997. Cell cycle-dependent establishment of a late replication program. Science 276: 806-809.

Ryba T, Hiratani I, Lu J, Itoh M, Kulik M, Zhang J, Schulz TC, Robins AJ, Dalton S, Gilbert DM. 2010. Evolutionarily conserved replication timing profiles predict long-range chromatin interactions and distinguish closely related cell types. Genome Res 20: 761-770.

Sadoni N, Cardoso MC, Stelzer EH, Leonhardt H, Zink D. 2004. Stable chromosomal units determine the spatial and temporal organization of DNA replication. J Cell Sci 117: 5353-5365.

Scharf AN, Barth TK, Imhof A. 2009. Establishment of histone modifications after chromatin assembly. Nucleic Acids Res 37: 5032-5040.

Smale ST, Fisher AG. 2002. Chromatin structure and gene regulation in the immune system. Annu Rev Immunol 20: 427-462.

Taylor JH. 1958. The mode of chromosome duplication in Crepis capillaris. Exp Cell Res 15: 350-357.

Taylor JH. 1960. Asynchronous duplication of chromosomes in cultured cells of Chinese hamster. J Biophys Biochem Cytol 7: 455-464.

Thurman RE, Day N, Noble WS, Stamatoyannopoulos JA. 2007. Identification of higher-order functional domains in the human ENCODE regions. Genome Res 17: 917-927.

van Bakel H, Nislow C, Biencowe BJ, Hughes TR. 2010. Most "dark matter" transcripts are associated with known genes. PLoS Biol 8: e1000371.

Venkatraman ES, Olshen AB. 2007. A faster circular binary segmentation algorithm for the analysis of array CGH data. Bioinformatics 23: 657-663.

Vogelauer M, Rubbi L, Lucas I, Brewer BJ, Grunstein M. 2002. Histone acetylation regulates the time of replication origin firing. Mol Cell 10: 1223-1233.

Walter J, Schermelleh L, Cremer M, Tashiro S, Cremer T. 2003. Chromosome order in HeLa cells changes during mitosis and early G1, but is stably maintained during subsequent interphase stages. J Cell Biol 160: 685-697.

Wu R, Terry AV, Singh PB, Gilbert DM. 2005. Differential subnuclear localization and replication timing of histone $\mathrm{H} 3$ lysine 9 methylation states. Mol Biol Cell 16: 2872-2881.

Wu R, Singh PB, Gilbert DM. 2006. Uncoupling global and finetuning replication timing determinants for mouse pericentric heterochromatin. J Cell Biol 174: 185-194.

Yokochi T, Poduch K, Ryba T, Lu J, Hiratani I, Tachibana M, Shinkai Y, Gilbert DM. 2009. G9a selectively represses a class of late-replicating genes at the nuclear periphery. Proc Natl Acad Sci 106: 19363-19368.

Zhou J, Deng Z, Norseen J, Lieberman PM. 2010. Regulation of Epstein-Barr virus origin of plasmid replication (OriP) by the S-phase checkpoint kinase Chk2. J Virol 84: 4979-4987. 


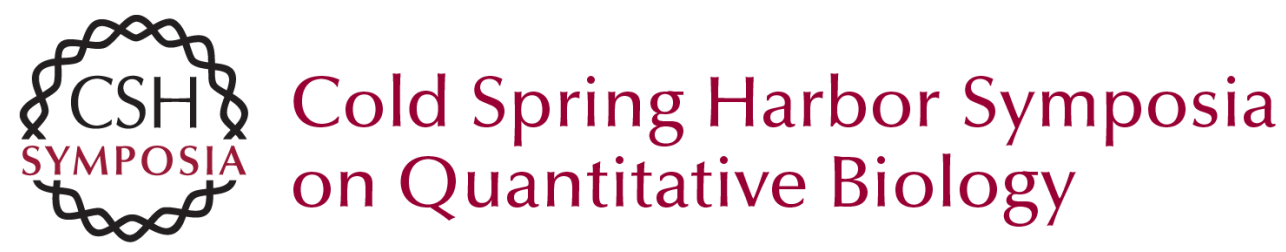

\section{Space and Time in the Nucleus: Developmental Control of Replication Timing and Chromosome Architecture}

D.M. Gilbert, S.-I. Takebayashi, T. Ryba, et al.

Cold Spring Harb Symp Quant Biol 2010 75: 143-153 originally published online December 7, 2010

Access the most recent version at doi:10.1101/sqb.2010.75.011

References This article cites 62 articles, 32 of which can be accessed free at: http://symposium.cshlp.org/content/75/143.full.html\#ref-list-1

\section{License}

Email Alerting Receive free email alerts when new articles cite this article - sign up in Service the box at the top right corner of the article or click here. 